\title{
Sistema informático para el análisis del comportamiento de los usuarios en Moodle
}

\section{Computer system for the analysis of user behavior in Moodle}

\section{Efraín Velasteguí López. ${ }^{1}$, Marco Antonio Gavilanes Sagñay. ${ }^{2}$ \& Willian Geovanny Yanza} Chávez. ${ }^{3}$

Recibido: 20-09-2019 / Revisado: 30-10-2019 /Aceptado: 24-11-2019/ Publicado: 07-12-2019

\begin{abstract}
DOI https://doi.org/10.33262/cienciadigital.v3i4.2.1026
\end{abstract}

With the technological advances of today, new techniques have been created for the development of distance learning and teaching, giving rise to the so-called e-learning. This platform proposes a new conception of education with the use of virtual environments based on the web as a tool to support the teaching-learning process. The emergence of specialized software has been encouraged, providing both teachers and students with an important variety of resources. However, despite the development of this software, there is still no system to analyze the preferences and learning trends of students in the Moodle learning management system. Therefore, it is necessary to develop a computer system that allows the study of user behavior in these platforms enabling students to have a more personalized service according to their needs and real interests. The development of this system will make it possible to improve the quality of education, offering students a more personalized service, which will boost their knowledge and thus facilitate learning through this type of platform.

Keywords: E-learning, Computer System, Moodle.

\section{Resumen}

Con los avances tecnológicos actuales, se han creado nuevas técnicas para el desarrollo de la enseñanza y aprendizaje a distancia, dando lugar al llamado e-learning. Esta plataforma

\footnotetext{
${ }^{1}$ Consorcio Ciencia Digital, Ambato, Ecuador, luisefrainvelastegui@ cienciadigital.org

${ }^{2}$ Magister en Interconectividad de Redes, ESPOCH, Riobamba, Ecuador, magavilanes@yahoo.es

${ }^{3}$ Magister en Informática Educativa, ESPOCH, Riobamba, Ecuador, williamyanza@ hotmail.com
} 
propone una nueva concepción en la educación con el uso de entornos virtuales basados en la web como una herramienta para apoyar el proceso de enseñanza - aprendizaje. Se ha fomentado la aparición de software especializado, que proporciona a los profesores y estudiantes una importante variedad de recursos; sin embargo, a pesar del desarrollo de este software todavía no existe un sistema para analizar las preferencias y tendencias de aprendizaje de los estudiantes. Por lo tanto, es necesario desarrollar un sistema informático que facilite el estudio del comportamiento del usuario en estas plataformas, permitiendo a los estudiantes tener un servicio personalizado, de acuerdo con sus necesidades e intereses reales. El desarrollo de este sistema permitirá mejorar la experiencia y calidad de la educación, ofreciendo a los estudiantes un servicio que contribuirá con sus conocimientos y facilitará el aprendizaje a través de este tipo de plataforma.

Palabras clave: E-learning, sistema informático, Moodle.

\section{Introducción}

El desarrollo de nuevas tecnologías de educación y comunicación ha hecho posible el uso de Internet y más específicamente la WWW (World Wide Web) en la educación a distancia, dando lugar a la llamada educación basada en la Web o e-learning (Horton, W. K., 2000).

En los últimos años, el e-learning implica una nueva concepción de la educación a través de la incorporación de nuevas tecnologías en el proceso de enseñanza y aprendizaje. Esta plataforma educativa proporciona a los docentes y estudiantes una importante variedad de recursos, que a menudo es una desventaja. Esto hace que el estudiante se desoriente ante la elección de los recursos diseñados. Este problema requiere una reestructuración de cómo el profesor ha diseñado la asignatura, con el objetivo de que este diseño se adapte mejor a las características e intereses de los estudiantes (Corso \& Alfaro, 2010).

El uso de entornos virtuales basados en la web, como una herramienta para apoyar el proceso educativo, se ha expandido en los últimos años, gracias al desarrollo de nuevos enfoques y modalidades de educación, como e-learning y b-learning. Estas nuevas formas de utilizar la tecnología como soporte para el proceso de enseñanza-aprendizaje han fomentado la aparición de software especializado en proporcionar los mecanismos que facilitan el desarrollo del trabajo autónomo del alumno, su interacción con el profesor y el uso de herramientas colaborativas y de comunicación. para trabajo en grupo (Aponte, Hoyos \& Monsalve, 2012).

Para ayudar a satisfacer estas necesidades, resolver este problema y mejorar la calidad de la educación superior en esta región a través de estas tecnologías, el Instituto Internacional para la Educación Superior en América Latina y el Caribe (IESALC) emprendió en 2002 un proyecto 
sobre educación superior virtual y a distancia. en esta región, que se insertó en su Programa macro llamado Observatorio de Educación Superior en América Latina y el Caribe (Silvio, 2004).

En varias universidades se han creado diferentes centros de estudio para la investigación en TIC para la educación superior, como la Facultad de Educación a Distancia de la Universidad de La Habana, el Centro de Referencia en Educación Avanzada (CREA), donde se encuentra la Universidad Virtual CUJAE, en España la UNIR expandió sus servicios educativos a varios países con la oferta de carreras y maestrías en modalidad virtual (Vázquez, 2008).

En la Universidad Agraria de La Habana, "Fructuoso Rodríguez Pérez" (UNAH) lleva varios años trabajando en la aplicación de tics a la Educación Superior. Expresiones de esto son la creación de un Laboratorio de Tecnología Educativa (LATED), donde se desarrolla una amplia variedad de software educativo. Pero a pesar de los grandes esfuerzos realizados para crear un sistema que permita estudiar el comportamiento de los estudiantes para permitir que el maestro pueda proporcionar al estudiante lo que realmente necesita para mejorar sus conocimientos con el uso de este tipo de tecnología.

Para aumentar el uso de estas plataformas, no existe un sistema que permita analizar las preferencias y tendencias que los estudiantes usan para estudiar, lo que permitiría mejorar la calidad de la educación, ofreciendo a los estudiantes un servicio personalizado de acuerdo con sus necesidades e intereses reales y de esta manera facilitan el aprendizaje a través de este tipo de plataforma en las universidades.

\section{Metodología}

La metodología de desarrollo de software utilizada para desarrollar este sistema informático fue el Proceso Racional Unificado (RUP). Este es un proceso de desarrollo de software que se adapta de acuerdo a las necesidades de los desarrolladores y el cliente, porque la comunicación entre ellos debe ser fluida, lo que permite llegar a un acuerdo para evitar futuros desacuerdos, además de integrar el Lenguaje Unificado de Modelado (UML) y es basado en componentes interconectados a través de una interfaz bien definida.

Con el propósito de ser temprano en la toma de decisiones, el análisis y la comprensión del comportamiento del usuario cobra una importancia fundamental, donde es necesario continuar en detalle sus acciones, lo que podría incluir toda la trayectoria de las páginas visitadas, el tiempo dedicado a cada uno de ellos, los productos elegidos, la cantidad de transiciones antes de terminar sumando una compra, etc. (Velásquez \& Palade, 2008). 
De esta manera, con el hecho de navegar en la Web se irá acumulando una serie de datos que brindarán excelente información: el registro web de registros, contienen todo el viaje de los usuarios; descargas asociadas (páginas, objetos multimedia, archivos, entre otros) y tiempo invertido (Ortega \& Aguillo, 2009).

Estos se generan cuando se realiza la lectura del código HTML de cada página que llega y es interpretado por el navegador, registrando cada objeto dado. Es importante indicar que, aunque solo se visita una página, más que un objeto se puede registrar, entre ellos una gran cantidad de objetos sin excelente información (banner publicitario, iconos, etc.), aunque esta relevancia dependerá en el estudio que se quiere realizar, por lo que filtrar y registrar los datos de interés previamente se convierte en una tarea clave (Velásquez \& Palade, 2008).

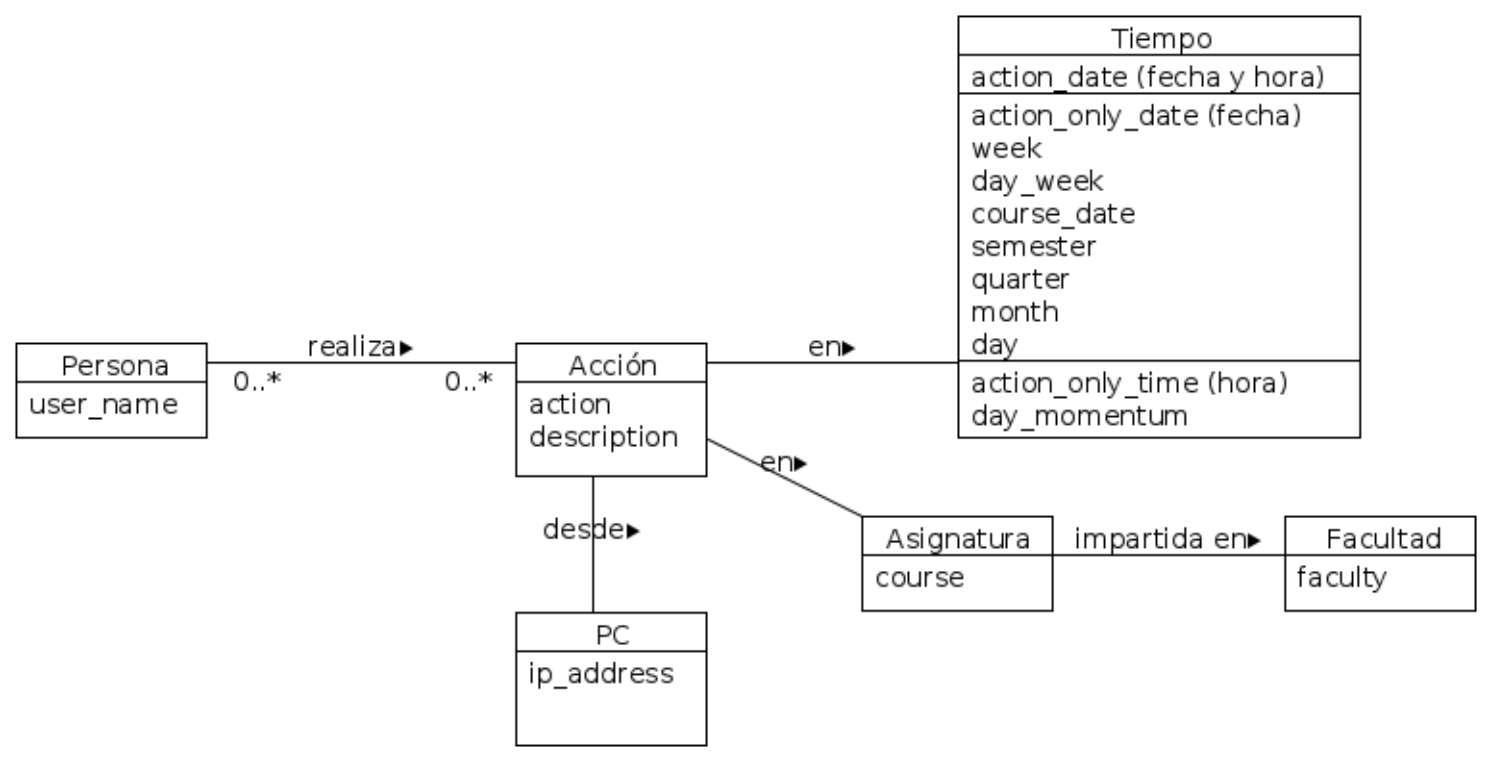

Figura 1: Diagrama de acciones

Fuente: Elaboración propia.

Para diseñar la base de datos, primero es necesario presentar el diagrama de clases persistentes con sus atributos y relaciones, y también es necesario aclarar que el marco Django tiene su propia base de datos, para lo que a continuación se muestra en las figuras 2.1 y 2.2, el patrón lógico y el patrón físico de los datos obtenidos, a partir de las entidades más significativas que se agregaron a la aplicación web. 


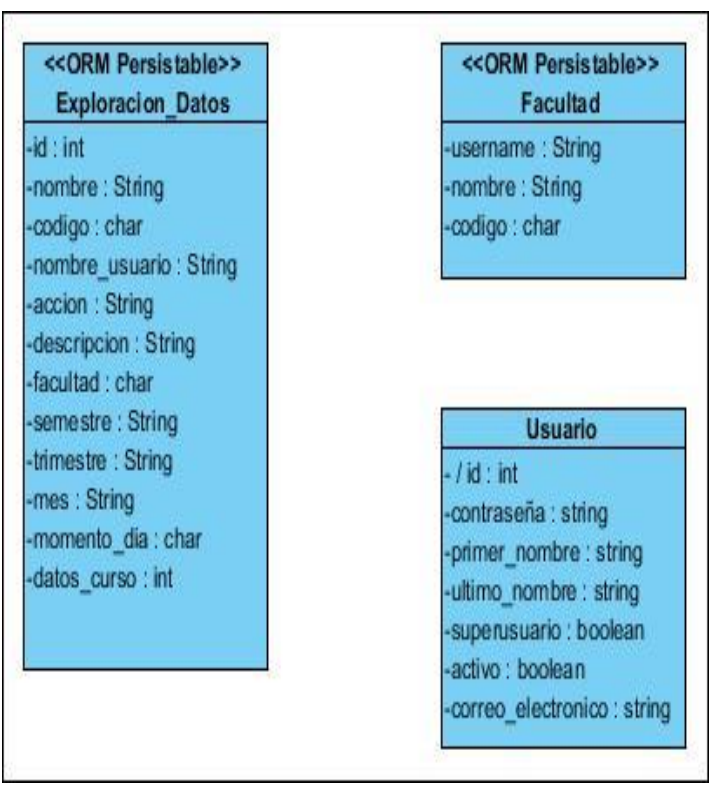

Figura 2.1: Modelo lógico de datos.

Fuente: Elaboración propia

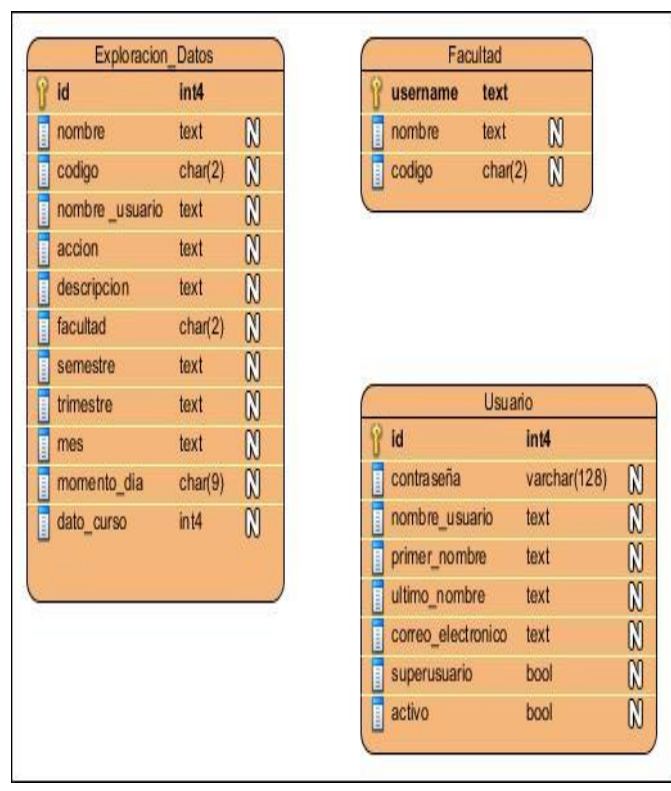

Figura 2.2: Modelo físico de datos.

Fuente: Elaboración propia

RUP se caracteriza por ser administrado por casos de uso, enfocados en arquitectura, iterativos e incrementales. Permite organizar las actividades de un equipo y dirige las tareas de cada desarrollador por separado y del equipo en su conjunto. Especifica los artefactos que deben desarrollarse, ofrece criterios para el control y medición de los productos (Carrasco, 2015).

Para el desarrollo del sistema informático, se utilizaron diferentes tecnologías como Java como lenguaje de programación, PostgreSQL como sistema de gestión de bases de datos, Visual Paradigm 8.0 como herramienta de casos, R y el marco MVC Django (Boza, 2016).

Django sigue al patrón MVC hasta el pie de la letra que un marco puede llamarse MVC. La M, V y C se separan en Django de la siguiente manera (Libros Web, 2016, El libro de Django 1.0) - M, la parte de acceso a la base de datos, es administrada por la capa de la base de datos de Django, que describiremos en este capítulo.

- V, la porción que selecciona qué datos mostrar y cómo mostrarlos, la vista y las plantillas lo manejan.

- El mismo marco que sigue su URLconf y llama a la función apropiada de Python para la URL obtenida administra $\mathrm{C}$, la porción que delega dependiendo de la entrada del usuario visible.

En el análisis de la información relacionada con el comportamiento del usuario en la plataforma de aprendizaje electrónico, se aplicaron estadísticas descriptivas utilizando un gráfico de barras para visualizar el uso de Moodle y estadísticas multivariadas a través del análisis de conglomerados para mostrar una agrupación de usuarios que tienen un comportamiento similar 
con respecto a los cursos que visitan y las acciones que realizan visualizándolo gráficamente (dendrograma).

\section{Resultados y discusión}

El sistema informático MoodleStat tiene una base de datos que proporcionada los registros de la plataforma Moodle para mostrar cómo se comportan los usuarios en esa plataforma con respecto a su acceso. Nos da en forma gráfica (histograma) cómo se comportan las facultades con respecto a los accesos de los usuarios a la plataforma en el año, por semestres, por trimestres, por meses y por cada momento del día. También muestra información general de las facultades en forma de tablas que ofrecen soporte para tener conocimiento y control de la plataforma, como el número de registros entre todas las facultades y para cada una de ellas (leer como un registro los accesos a la plataforma de usuarios) la fecha del primer y último registro, el número total de usuarios que se han conectado entre todas las facultades y para cada una de ellas, el número de cursos que existen en cada facultad y los más visitados entre todas las facultades, acciones realizado por los usuarios en esos cursos y el más utilizado entre todas las facultades, los usuarios que más acceden a la plataforma y el número total de veces que el usuario ha visitado un curso por cada curso visitado en el año en que lo visitó. Además mostrará una agrupación por usuarios que tienen un comportamiento similar con respecto a los cursos que visitan y las acciones realizadas visualizándolo gráficamente (dendrograma) y mostrando a los subgrupos separados de los usuarios los temas y acciones con comportamiento similar en forma de barra gráfico (histograma).

En primer lugar, el sistema muestra una interfaz para que el usuario introduzca su nombre y firma (ver figura 3) para poder ver y analizar los datos que muestra el sistema. Este sistema utiliza dos listas en su funcionamiento: el administrador del sistema y el otro es el profesor.

El administrador del sistema se encarga de la administración de los usuarios, de las capacidades y de agregar nuevos registros de la base de datos de Moodle para modernizar la información que se muestra en la aplicación. El profesor tendrá acceso total a la información que el sistema muestra que los usuarios se comportan en la plataforma de acuerdo con sus accesos sobre cómo, los cursos que visitan y las acciones que realizan en ellos. 


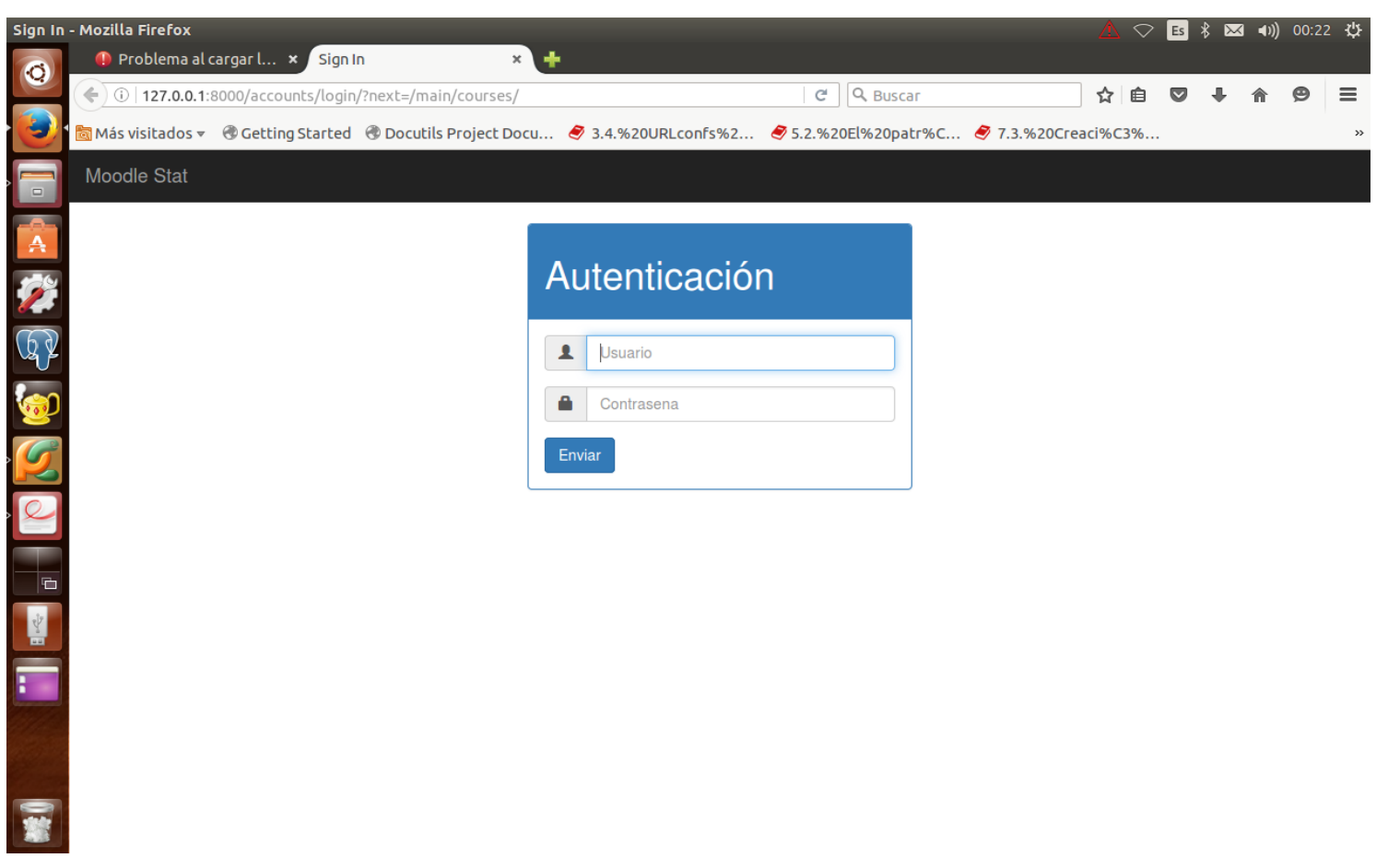

Figura 3: Interfaz de autenticación del sistema informático.

Fuente: Elaboración propia

La Figura 4 muestra la interfaz relacionada con el análisis entre todas las facultades, los accesos para cada una de las asignaturas y un gráfico de barras que representa el comportamiento de los usuarios según su acceso a la plataforma en cada una de las asignaturas.

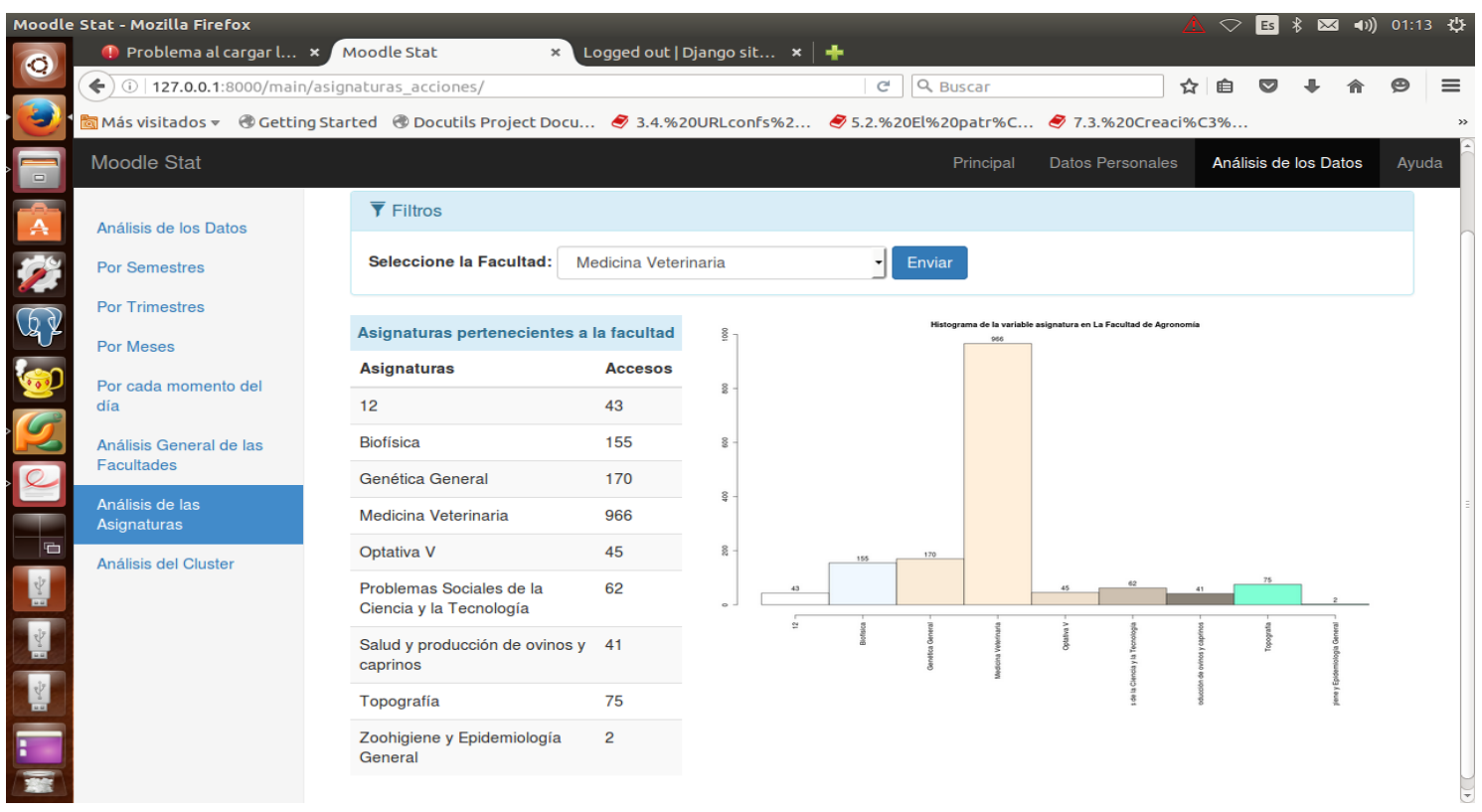

Figura 4: Interfaz de análisis de los sujetos.

Fuente: Elaboración propia 
El análisis del comportamiento de los meses se visualiza en la figura 5, donde se destaca que los meses convergentes son octubre y noviembre, siendo este último mes donde se realizan los exámenes intersemestrales y trabaja extra clases antes de terminar el semestre.

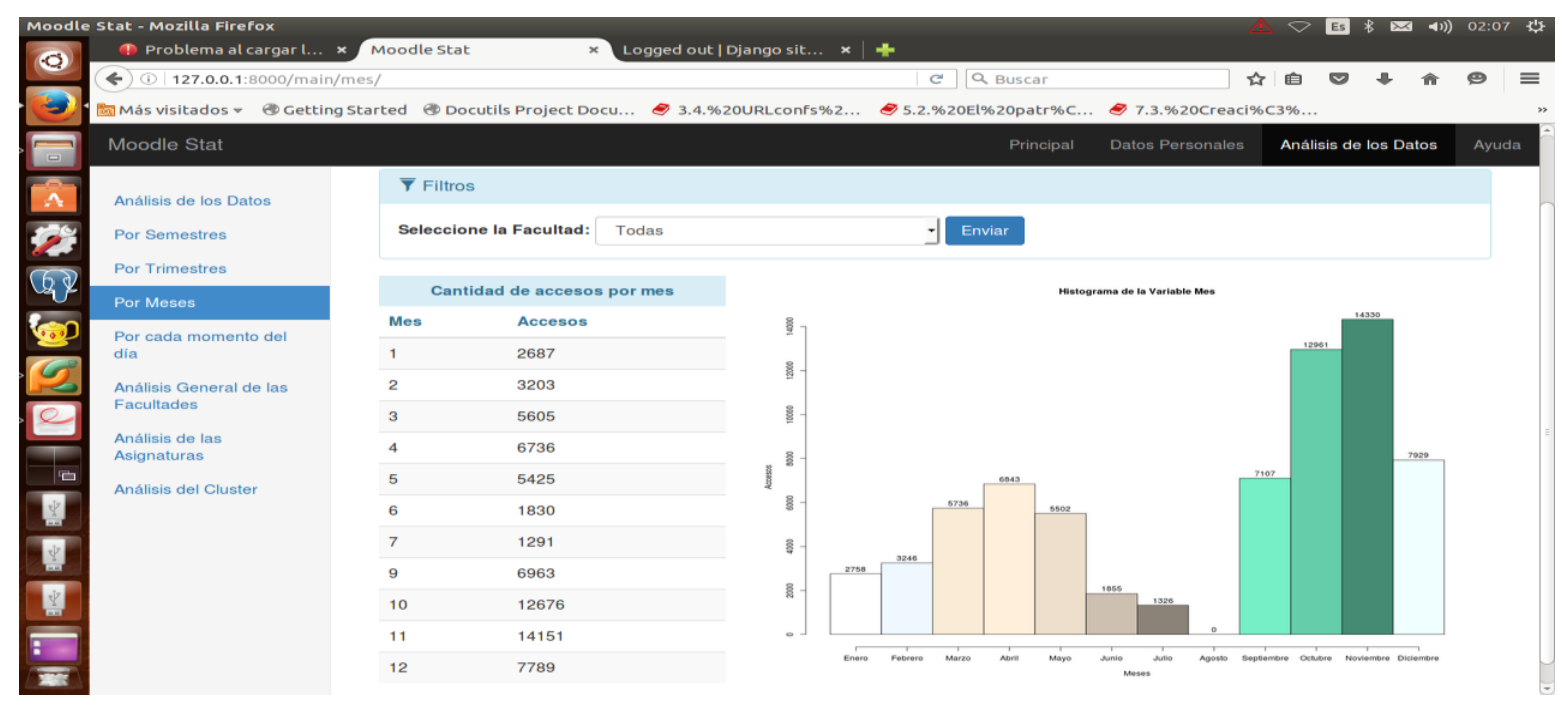

Figura 5: Comportamientos de los accesos por meses.

Fuente: Elaboración propia

En la figura 6 se muestra el comportamiento de los accesos de los usuarios por momentos del día, siendo la mañana el momento del día más concurrido por los alumnos y profesores.

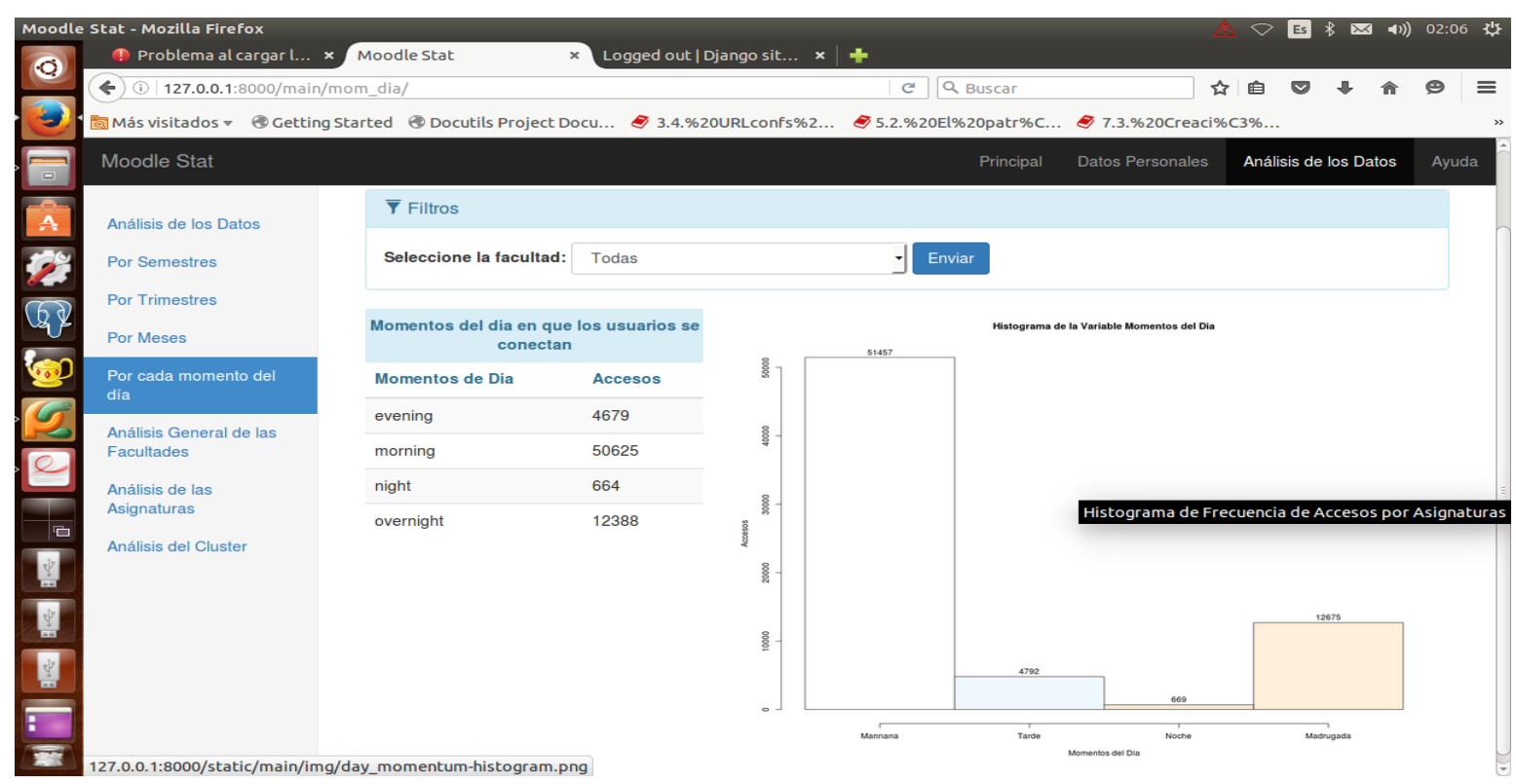

Figura 6: Comportamientos de los accesos para momentos del día.

Fuente: Elaboración propia 
Otro resultado interesante se visualiza en la figura 7, en la misma que llevas a la agrupación de usuarios según sus comportamientos con respecto a los cursos que visitan y las acciones que se llevan a cabo en ellos, mostrando un dendrograma para los usuarios filtrados en a formar sus acciones y los grupos formados por cada uno de sus miembros.

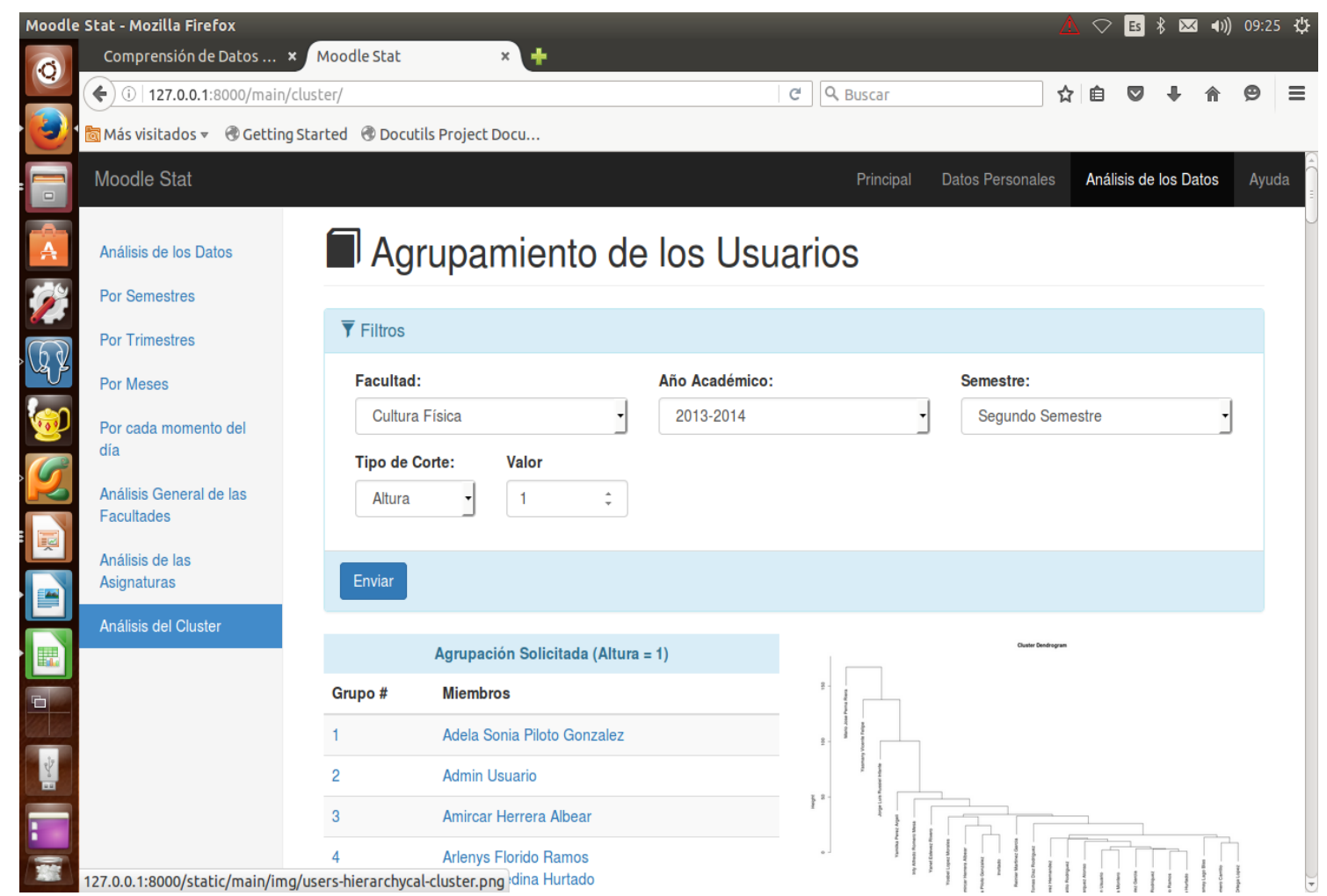

Figura 7: Agrupación de los usuarios

Fuente: Elaboración propia

\section{Conclusiones}

La realización del software permite el estudio de los perfiles de usuario con respecto al uso de la plataforma Moodle y su incorporación proporcionará información sobre el comportamiento de los usuarios de acuerdo con su acceso y las acciones en la plataforma Moodle.

Al poder establecer estadísticas de preferencia de cursos y poder segmentar los usuarios por categorías demográficas como son: edades, sexo, estudios, etc, será posible una mejor programación de los cursos y un eficiente uso de los recursos.

El conocer los cursos con mayor demanda, establece una clara guía del mercado educativo sobre la tendencia de lo que el mercado exige de sus colaboradores, que es precisamente en los que se capacitan con mayor frecuencia. 


\section{Referencias Bibliográficas:}

Aponte, F. A., Hoyos, J. G. \& Monsalve, J. A. (2012). Minería de usabilidad aplicada a plataformas virtuales de aprendizaje. Revista Virtual Universidad Católica del Norte, núm. 37, septiembre-diciembre, pp. 27-43.

Boza, J.C. (2016). Sistema Informático para el análisis de los perfiles de usuarios en plataforma e-learning de la UNAH. Tesis presentada en opción al Título de Ingeniero en Informática. Universidad Agraria de la Habana. Provincia Mayabeque.80p.

Carrasco, A. F. (2015). Módulo Gestión de Proyectos Internacionales para el Sistema de Información Gerencial del Centro Nacional de Sanidad Agropecuaria.

Corso, C. L. \& Alfaro, S. L. (2010). Minería de uso web: Presentación de caso práctico para el mejoramiento de la Educación, basada en el uso de plataformas virtuales. XII Workshop de Investigadores en Ciencias de la Computación, p.177-182.

Horton, W. K. (2000). Designing web-based training: how to teach anyone anything anywhere anytime. ISBN-10:047135614XNew York.

Libros Web (2016). El libro de Django 1.0 . Disponible en: www.http://librosweb.es

Ortega-Priego, J.-L. \& Aguillo, I. F (2009). Minería del uso de webs. El profesional de la información, 18, 20-26.

Silvio, J. (2004). Tendencias de la educación superior virtual en América Latina y el Caribe.

Publicaciones UNESCO-IESALC. México, D. F, 237 p

Vázquez, Y. (2008). Sistema de objetos de aprendizaje para la enseñanza de la Matemática básica en la carrera Ingeniería Agronómica. Tesis en opción al grado de Master en Nuevas Tecnologías, Universidad Agraria de la Habana.100p.

Velásquez, J and Palade, V (2008). Adaptive web sites: A knowledge extraction from web data approach. Proceedings of the 2008 conference on Adaptive Web Sites: A Knowledge Extraction from Web Data Approach. IOS Press, 1-272.

\section{\Ciencia}




\section{PARA CITAR EL ARTÍCULO INDEXADO.}

Velasteguí López, E., Gavilanes Sagñay, M., \& Yanza Chávez, W. (2019). Sistema informático para el análisis del comportamiento de los usuarios en Moodle. Ciencia Digital, 3(4.2), 217-227. https://doi.org/10.33262/cienciadigital.v3i4.2.1026

\section{Ligital}

El artículo que se publica es de exclusiva responsabilidad de los autores y no necesariamente reflejan el pensamiento de la Revista Ciencia Digital.

El artículo queda en propiedad de la revista y, por tanto, su publicación parcial y/o total en otro medio tiene que ser autorizado por el director de la Revista Ciencia Digital.
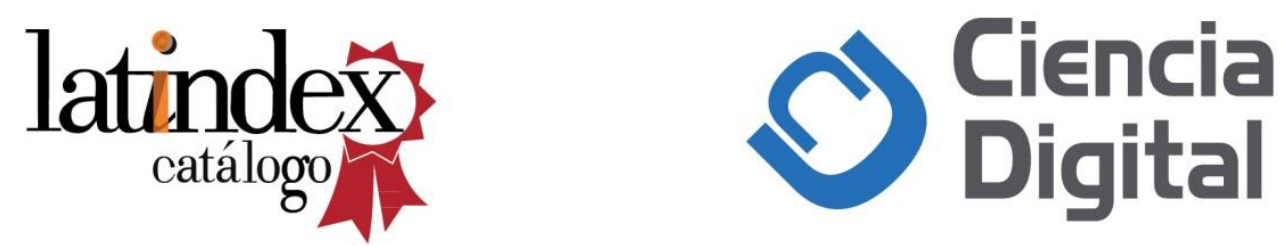